\title{
Tackling vaccine refusal
}

\author{
Bert Gordijn ${ }^{1} \cdot$ Henk ten Have ${ }^{2}$
}

Published online: 2 February 2022

(C) The Author(s), under exclusive licence to Springer Nature B.V. 2022

The COVID-19 pandemic has demonstrated that developing and producing the right technical tools to combat the disease such as vaccines, test kits and masks might not suffice to guarantee success. Even when the tools are produced in sufficient numbers, and the logistics and pricing are such that they are readily available and affordable for everyone, if people are not willing to use those tools, they are not going to be effective. This circumstance points to an important public health policy challenge, i.e., to develop effective means to increase public uptake of the tools deemed necessary to fight a disease. This challenge has increasingly become the focus of much political and public discussion.

\section{Stigmatization}

In the first paper of the current issue Kärki (2022) argues that ethical condemnation of vaccine refusal is not the most effective means to enhance vaccine uptake. In fact, labeling vaccine refusers as free riders who intend to enjoy the benefits of herd immunity without being willing to bear any of the burdens themselves is a mistake.

\section{Motivations}

Kärki (2022) explains that social science research has cast serious doubt on the idea of free riding on herd immunity as the factual motivation of vaccine refusers. For one, intentional free riding is subverted by the dissemination of antivaccination material, and yet, vaccine refusers frequently engage in it. In fact, free riding does not seem to play a substantial role in motivating vaccine refusal. Instead, refusal is most often motivated by other factors such as negative

\footnotetext{
Bert Gordijn

bert.gordijn@dcu.ie

Dublin City University, Dublin, Ireland

2 Duquesne University, Pittsburgh, PA, USA
}

experiences with healthcare workers, observed injustices within the healthcare system, distrust of healthcare institutions, and distance from the political establishment (Kärki 2022). For a better conceptual analysis of these motivations Kärki (2022) deploys Hirschman's (1970) ideas of exit and voice, whereby citizens' frustration with an institution is either articulated, by voice, or in case this is deemed ineffective, by exit, i.e., by giving up on the institution altogether. "If citizens have lost trust in the effectivity of public outcries, they may turn to more invisible forms of opposing policies" (Kärki 2022). In these terms, Kärki (2022) argues that vaccine refusal could be regarded as an endeavour to exit the public good of herd immunity.

\section{Solutions}

If vaccine refusal is solely understood from a specific normative angle whereby it is denounced as free riding, sanctions may seem appropriate. However, based on her analysis of the factual motivations of vaccine refusal, Kärki (2022) is concerned that stigmatization and attempts to enforce compliance might generate further polarization instead of convincing vaccine refusers to change their ways. Vaccine mandates run the risk of enhancing distrust and grievances (Kärki 2022). Instead, if hesitancy and refusal are to be understood in terms of an exit dynamic, it is necessary to improve the communication and information channels to better hear and address the concerns of the vaccine refusers. Overall, we need to conduct better and more empirical research to develop improved approaches to counter vaccine refusal with success (Kärki 2022).

\section{A call for social science research}

Without commenting on the correctness of the details of Kärki's (2022) analysis, her focus on the factual motivations for vaccine refusal and her call for empirical research with a view to develop effective means to address it are spot 
on. The lack of COVID-19 vaccine uptake, which currently curbs the effectiveness of public health policies in a range of countries, clearly demonstrates the limits of a technofix approach to public health. Thus, more social science research is needed into the roots of vaccine refusal. Compared to the high-level sophistication of our scientific understanding of COVID-19 and the various medical tools to fight the disease, our scientific comprehension of the psychological and sociological roots of vaccine acceptance, hesitancy and refusal is lagging. This should urgently be addressed.

In addition to social science research and informed by its results, it is important to develop a more subtle approach to normative messaging. Especially when the messaging is meant to accomplish behavioural change, a constructive message that encourages solidarity and appeals to responsibility might be more effective than a normative approach that is exclusively condemnatory and punitive.

\section{References}

Hirschman, Albert O. 1970. Exit, voice and loyalty: Responses to decline in firms, organizations and states. Cambridge, MA: Harvard University Press.

Kärki, K. 2022. Listening to vaccine refusers. Medicine, Health Care and Philosophy. https://doi.org/10.1007/s11019-021-10055-y.

Publisher's Note Springer Nature remains neutral with regard to jurisdictional claims in published maps and institutional affiliations. 